\title{
On Boundary Value Problems for Elliptic Equations Degenerating on the Boundary
}

\author{
By \\ Akira NAKAOKA*
}

\section{Introduction}

In this paper we consider the equation of the following form;

$$
-\rho(x) L(x, D) u+\sum_{j=1}^{n} p_{j}(x) \frac{\partial u}{\partial x_{j}}+q(x) u+\lambda u=f(x)
$$

in $\Omega \subset R^{n}$, where $\Omega$ is an interior or an exterior of a smooth and compact hypersurface, $\lambda$ is a complex number and $\rho(x)$ is a real-valued function satisfying

(1) $\rho(x)$ is continuous and $0 \leqq \rho(x) \leqq M$ on $\bar{\Omega}$

(2) $\rho(x)$ vanishes only on the boundary of $\Omega$, which we denote it by $\Gamma$

(3) $\rho(x)$ depends only on $r=r(x)$, the distance from $x \in \bar{\Omega}$ to $\Gamma$ in some neighbourhood of $\Gamma$

(4) for small $r$, there exist two constants $A_{1}$ and $A_{2}$ such that $A_{1} r^{\alpha} \leqq \rho(x) \leqq A_{2} r^{\alpha} \quad(0<\alpha<1)$

(5) when $\Omega$ is unbounded, $0<K \leqq \rho(x)$ as $|x| \rightarrow \infty$.

$L(x, D)$ is an elliptic operator of the form

$$
L(x, D)=\sum_{j, k=1}^{n} \frac{\partial}{\partial x_{j}}\left(a_{j k}(x) \frac{\partial}{\partial x_{k}}\right)+\sum_{j=1}^{n} b_{j}(x) \frac{\partial}{\partial x_{j}}+c(x)
$$

with $a_{j k}(x)$ real symmetric and

Received May 7, 1971.

Communicated by S. Matsuura.

* Department of Mathematics, Ritsumeikan University, Kinugasa, Kyoto, Japan. 


$$
c_{1}|\xi|^{2} \leqq \sum_{j, k=1}^{n} a_{j k}(x) \xi_{j} \xi_{k} \leqq c_{2}|\xi|^{2}
$$

for any $x \in \bar{\Omega}$ and for any $\xi=\left(\xi_{1}, \ldots, \xi_{n}\right) \in R^{n}$.

For the regularity of the coefficients we assume that $a_{j k}(x)$ 's are all in $\mathscr{B}^{1}(\Omega)$ and $b_{j}(x)$ 's, $p_{j}(x)$ 's, $c(x)$ and $q(x)$ are all bounded and measurable.

We impose the Dirichlet or Neumann condition as boundary condition, that is, we seek the solution satisfying

$$
\left.u\right|_{\Gamma}=\phi
$$

or

$$
\frac{\partial u}{\partial \nu}+\left.\sigma u\right|_{r}=\phi
$$

where

$$
\frac{\partial}{\partial \nu}=\sum_{j, k=1}^{n} a_{j k}(x) \nu_{j} \frac{\partial}{\partial x_{k}}
$$

with $\nu=\left(\nu_{1}, \ldots, \nu_{n}\right)$ the unit outer normal at each boundary point.

The problem with homogeneous Dirichlet condition was treated in [6] under the slightly different conditions on $\rho(x)$.

We study our problems through the Hilbert space methods. As was remarked in [7], it is much important to settle suitable function spaces in treating the degenerate boundary value problems. In our case, the following space will be fittable as a basic space.

Definition 1.1. We denote by $L^{2}\left(\Omega, \rho^{-1}\right)$ the totality of the functions on $\Omega$ satisfying

$$
\|u\|_{0, \rho^{-1}}^{2}=\int_{\Omega} \frac{|u(x)|^{2}}{\rho(x)} d x<+\infty
$$

Instead of usual Sobolev space $H^{m}(\Omega)$, we introduce the weighted Sobolev space $H^{m}(\Omega, \rho)$ by

Definition 1.2. We denote by $H^{m}(\Omega, \rho)$ the totality of the functions on $\Omega$ satisfying 


$$
\|\left. u\right|_{m, \rho} ^{2}=\int_{\Omega}\left(\rho(x) \sum_{\mid \alpha_{1}=m}\left|D^{\alpha} u\right|^{2}+|u|^{2}\right) d x<+\infty
$$

It can be easily seen that $L^{2}\left(\Omega, \rho^{-1}\right)$ and $H^{m}(\Omega, \rho)(m=0,1, \ldots)$ make Hilbert spaces by their norm.

Our main aim is to show

Theorem 1.1. For any $f(x) \in L^{2}(\Omega, \rho)$ and for any $\phi \in H^{\beta}(\Gamma)$, there exists a real number $\lambda_{0}$ such that for all $\lambda \geqq \lambda_{0}$, the equation (1.1) has a unique solution $u(x)$ belonging to $H^{2}(\Omega, \rho)$, where

$$
\beta= \begin{cases}\frac{3-\alpha}{2} & \text { (Dirichlet problem) } \\ \frac{1-\alpha}{2} & \text { (Neumann problem) }\end{cases}
$$

and $\sigma \in \mathscr{B}^{1}(\Gamma)$.

From the point of view of mathematical physics, our equation will be related with the following situation: for example, consider the wave equation

$$
\mu(x) \frac{\partial^{2} u}{\partial t^{2}}=\Delta u+f(x, t)
$$

where $\mu(x)$ denotes the density of medium. Dividing both sides of (1.6) by $\mu(x)$, we obtain

$$
\frac{\partial^{2} u}{\partial t^{2}}=\rho(x) \Delta u+g(x, t)
$$

with $\rho(x)=1 / \mu(x)$ and $g(x, t)=\rho(x) f(x, t)$. And our equation corresponds to the case of steady state in (1.7) when $\mu(x)$ becomes infinitely large on the boundary. The corresponding heat equation can also be treated.

Utilizing our results, we can show the well-posedness of the mixed problems for (1.6) (of course for rather general equations) with Dirichlet or Neumann condition (see Appendix).

\section{Some Properties of $H^{m}(\Omega, \rho)$ and Some Estimates}

In this section we prepare a few theorems stating the properties of 
$H^{m}(\Omega, \rho)$ and some basic estimates. One of our interest is of the imbedding of $H^{m}(\Omega, \rho)$ into a certain usual Sobolev space, which will be of fractional order, and another is the trace of the member of $H^{m}(\Omega, \rho)$ to a submanifold of $\Omega$. For these questions we can refer many studies by Soviet mathematicians, for example, S.V. Uspenskii [9], [10], and P.I. Lizorkin [3], [4].

The following is a special case of the theorem due to Uspenskii and Lizorkin (see S.M. Nikol'skii [8] also).

Theorem 2.1. The following continuous imbedding holds;

$$
H^{m}(\Omega, \rho) \rightarrow H^{m-\alpha / 2}(\Omega) \rightarrow H^{m-(\alpha+1) / 2}(\Gamma),
$$

where the second arrow is the trace operator.

Corollary 2.1. Let $u(x)$ be in $H^{m}(\Omega, \rho)$, then for any $\varepsilon>0$ it holds

$$
\left|\gamma D^{\alpha} u\right|_{0} \leqq \varepsilon\|u\|_{m, \rho}+C(\varepsilon)\|u\| \quad(|\alpha| \leqq m-1),
$$

where $\gamma$ denotes the trace operator to $\Gamma,||_{0}$ the $L^{2}(\Gamma)$ norm and \|\| the $L^{2}(\Omega)$ norm.

The following theorem is much important for us to reduce our problems to the case of homogeneous boundary condition.

Theorem 2.2. For any $\phi \in H^{(3-\alpha) / 2}(\Gamma)$ there exists a function $u(x)$ in $H^{2}(\Omega, \rho)$ such that

$$
\left.u\right|_{\Gamma}=\phi
$$

Proof. It will be sufficient to show the assertion when $\Omega$ is $\mathbf{R}_{+}^{n}=\left\{(x, y) ; x>0\right.$ and $\left.y \in R^{n-1}\right\}$ after localizing this problem by partition of unity.

Now let us define $u(x, y)$ by

$$
u(x, y)=\int_{\mathbf{R}^{n-1}} \hat{\phi}(\eta) e^{-(1+|\eta|) x} e^{2 \pi i y \cdot \eta} d \eta
$$

then we can easily verify that $u(x, y)$ gives a function satisfying the assertion, where ^ means the tangential Fourier transformation. Q.E.D. 
For the Neumann problem, we have

Theorem 2.3. For any $\phi \in H^{(1-\alpha) / 2}(\Gamma)$ and for any first order differential operator $B(x, D)$ with smooth bounded coefficients, which is transversal on $\Gamma$, there exists a function $u(x)$ in $H^{2}(\Omega, \rho)$ such that

$$
\left.B(x, D) u\right|_{\Gamma}=\phi .
$$

Proof. Assume $\Omega=\mathbf{R}_{+}^{n}$ and then $B(x, D)$ is of the form

$$
B=\frac{\partial}{\partial x}+\sum_{j=1}^{n-1} a_{j}(y) \frac{\partial}{\partial y_{j}}+b(y)
$$

Now define $u(x, y)$ by

$$
u(x, y)=x \int_{\mathbf{R}^{n-1}} \hat{\phi}(\eta) e^{-(1+|\eta|) x} e^{2 \pi i y \cdot \eta} d \eta
$$

then it gives our demanded one.

Q.E.D.

The following will be useful.

Proposition 2.1. Let $u(x)$ be in $H^{m}(\Omega, \rho)$, then for any $\varepsilon>0$ it holds

$$
\int_{\Omega} \frac{\left|D^{\alpha} u\right|^{2}}{\rho(x)} d x \leqq \varepsilon\|u\|_{m, \rho}^{2}+C(\varepsilon)\|u\|^{2}
$$

with $|\alpha| \leqq m-1$.

Proof. It will be sufficient to show when $\Omega=\mathbf{R}_{+}^{n}$ and $|\alpha|=m-1$. Moreover we may assume $\rho(x)$ is a function of only normal argument.

Note

$$
\left(D^{\alpha} u\right)(x, y)=\left(D^{\alpha} u\right)(0, y)+\int_{0}^{x} \frac{\partial}{\partial \xi}\left(D^{\alpha} u\right)(\xi, y) d \xi
$$

for almost every $y$, then we have

$$
\left|\left(D^{\alpha} u\right)(x, y)\right|^{2} \leqq 2\left(\left|\left(D^{\alpha} u\right)(0, y)\right|^{2}+\int_{0}^{x} \frac{d \xi}{\rho(\xi)} \int_{0}^{\infty} \rho(x) \sum_{|\alpha|=m}\left|D^{\alpha} u\right|^{2} d x\right) .
$$

Thus for any $\delta>0$, we obtain 


$$
\int_{0}^{\delta} \frac{\left|D^{\alpha} u\right|^{2}}{\rho(x)} d x \leqq K(\delta)\left(\left|\left(D^{\alpha} u\right)(0, y)\right|^{2}+\int_{0}^{\infty} \rho(x) \sum_{|\alpha|=m}\left|D^{\alpha} u\right|^{2} d x\right)
$$

with $K(\delta) \rightarrow 0$ as $\delta \rightarrow 0$, and hence

$$
\int_{\mathbf{R}^{n-1}} \int_{0}^{\delta} \frac{\left|D^{\alpha} u\right|^{2}}{\rho(x)} d x d y \leqq K(\delta)\left(\left|\left(D^{\alpha} u\right)(0, \cdot)\right|_{0}^{2}+\|u\|_{m, \rho}^{2}\right) .
$$

On the other hand, we get by Theorem 2.1,

$$
\int_{\mathbf{R}^{n-1}} \int_{\delta}^{\infty} \frac{\left|D^{\alpha} u\right|^{2}}{\rho(x)} d x d y \leqq A(\delta)\|u\|_{m-1}^{2}
$$

and hence

$$
\leqq A(\delta)\left(\varepsilon^{\prime}\|u\|_{m, \rho}^{2}+C\left(\varepsilon^{\prime}\right)\|u\|^{2}\right)
$$

where $\varepsilon^{\prime}$ is an arbitrary positive number and

$$
A(\delta)=\sup _{x \geqq \delta} \frac{1}{\rho(x)}
$$

Hence we obtain

$$
\begin{gathered}
\int_{\mathbf{R}_{+}^{n}} \frac{\left|D^{\alpha} u\right|^{2}}{\rho(x)} d x d y \leqq K(\delta)\left|\left(D^{\alpha} u\right)(0, \cdot)\right|_{0}^{2}+\left(K(\delta)+A(\delta) \varepsilon^{\prime}\right)\|u\|_{m, \rho}^{2}+ \\
+A(\delta) C\left(\varepsilon^{\prime}\right)\|u\|^{2}
\end{gathered}
$$

and using Corollary 2.1, (or rather Theorem 2.1 again), and choosing $\delta$ and $\varepsilon^{\prime}$ suitably, we can get (2.2).

Q.E.D.

\section{A priori Estimates of the Solutions \\ of the Special Cases in a Half-Space}

Here we derive a priori estimates of the solutions of the boundary value problem for the special equation in a half-space. We assume that the weight function depends only on the normal argument $x$ through this section.

We consider first Dirichlet problem for the following equation; 


$$
-\rho(x)\left(\frac{\partial^{2} u}{\partial x^{2}}+2 \sum_{j=1}^{n-1} a_{j} \frac{\partial^{2} u}{\partial x \partial y_{j}}+\sum_{j, k=1}^{n-1} b_{j k} \frac{\partial^{2} u}{\partial y_{j} \partial y_{k}}-u\right)=f(x, y)
$$

with boundary condition

$$
\left.u(x, y)\right|_{x=0}=\phi(y)
$$

where $a_{j}$ 's and $b_{j k}$ 's $\left(b_{j k}=b_{k j}\right)$ are all real constants such that

$$
\xi^{2}+2 \sum_{j=1}^{n-1} a_{j} \xi \eta_{j}+\sum_{j, k=1}^{n-1} b_{j k} \eta_{j} \eta_{k} \geqq c\left(\xi^{2}+|\eta|^{2}\right)
$$

for any $(\xi, \eta) \in \mathbf{R}^{1} \times \mathbf{R}^{n-1}$. We assume moreover

$$
\int_{\mathbf{R}_{+}^{n}} \frac{|f(x, y)|^{2}}{\rho(x)} d x d y<+\infty
$$

and $\phi(y)$ belongs to $H^{(3-\alpha) / 2}\left(\mathbf{R}^{n-1}\right)$.

Let us denote by $\hat{u}(x, \eta)$ the tangential Fourier image of $u(x, y)$ :

$$
\hat{u}(x, \eta)=\int_{\mathbf{R}^{n-1}} u(x, y) e^{-i y \cdot \eta} d y .
$$

After the tangential Fourier transformation, the equation (3.1) turns into

$$
-\rho(x)\left(\frac{d^{2} \hat{u}}{d x^{2}}-2 i\left(\sum_{j=1}^{n-1} a_{j} \eta_{j}\right) \frac{d \hat{u}}{d x}-\left(\sum_{j, k=1}^{n-1} b_{j k} \eta_{j} \eta_{k}\right) \hat{u}-\hat{u}\right)=\hat{f}(x, \eta)
$$

and the boundary condition into

$$
\left.\hat{u}(x, \eta)\right|_{x=0}=\hat{\phi}(\eta)
$$

We shall seek a solution $\hat{u}(x, \eta)$ (with parameter $\eta$ ) belonging to $L^{2}\left(\mathbb{R}_{+}^{1}\right)$ satisfying (3.4) and (3.5).

Now let $\theta(\eta)$ be the root with negative real part of the following algebraic equation in $\theta$

$$
\theta^{2}-2 i\left(\sum_{j=1}^{n-1} a_{j} \eta_{j}\right) \theta-\left(\sum_{j, k=1}^{n-1} b_{j k} \eta_{j} \eta_{k}+1\right)=0
$$

$\theta(\eta)$ is given by 


$$
\theta(\eta)=i\left(\sum_{j=1}^{n-1} a_{j} \eta_{j}\right)-\sqrt{\sum_{j, k=1}^{n-1} b_{j k} \eta_{j} \eta_{k}+1-\left(\sum_{j-1}^{n-1} a_{j} \eta_{j}\right)^{2}}
$$

Let us remark it follows

$$
\sum_{j, k=1}^{n-1} b_{j k} \eta_{j} \eta_{k}+1-\left(\sum_{j-1}^{n-1} a_{j} \eta_{j}\right)^{2} \geqq c^{\prime}(1+|\eta|)^{2} .
$$

In fact taking $\xi=-\left(\sum_{j=1}^{n-1} a_{j} \eta_{j}\right)$ in (3.3), we can easily obtain (3.6).

Now in order to obtain a priori estimates of the solutions, we prepare the following.

Proposition 3.1. Let us define an integral operator $K_{\delta}$ by

$$
\begin{array}{r}
\left(K_{\delta} f\right)(x)=\delta \int_{0}^{\infty} e^{-\delta|x-y|}\left(\frac{x}{y}\right)^{\alpha / 2} f(y) d y \\
(-1<\alpha<1 \quad \text { and } \delta>0)
\end{array}
$$

then $K_{\delta}$ is a bounded operator from $L^{2}(0, \infty)$ to $L^{2}(0, \infty)$ and its operator norm is uniformly bounded in $\delta$.

Proof. It will sufices to show when $0 \leqq \alpha<1$ and then to show for any $f(x)$ and $g(x)$ in $L^{2}(0, \infty)$

$$
J=\left|\int_{0}^{\infty} \int_{0}^{\infty} \delta e^{-\delta|x-y|}\left(\frac{x}{y}\right)^{\alpha / 2} f(y) \overline{g(x)} d y d x\right| \leqq C\|f\|\|g\| .
$$

Now let us divide the quarter plane $\{(x, y) ; x, y>0\}$ into two parts;

1) $|x-y| \leqq(x+y) / 2$

2) $|x-y|>(x+y) / 2$.

In the case of 1$)$, since $x / y \leqq 3$, it follows immediately

$$
J \leqq 3^{\alpha / 2} \int_{0}^{\infty} \int_{0}^{\infty} \delta e^{-\delta|x-y|}|f(y)||g(x)| d y d x \leqq 3^{\alpha / 2}\|f\|\|g\| .
$$

In the case of 2 ), since $e^{-\delta ! x-y \mid}<e^{-\delta x / 2} e^{-\delta y / 2}$, it follows

$$
\begin{aligned}
& J \leqq \int_{0}^{\infty} \sqrt{\delta} e^{-\delta x / 2} x^{\alpha / 2}|g(x)| d x \int_{0}^{\infty} \sqrt{\delta} e^{-\delta y / 2} y^{-\alpha / 2}|f(y)| d y \\
& \leqq \sqrt{\Gamma(1+\alpha) \Gamma(1-\alpha)}\|f\|\|g\| .
\end{aligned}
$$


Thus (3.7) is correct.

Q.E.D.

Now let us establish a priori estimates for $u(x, y)$ satisfying (3.4) and (3.5). We note that $u(x, y)$ satisfying (3.4) and (3.5) can be expressed as

$$
\begin{aligned}
& \hat{u}(x, \eta)=\frac{1}{2 \theta(\eta)} \int_{0}^{\infty} e^{\theta(\eta)|x-\xi|} \frac{\hat{f}(\xi, \eta)}{\rho(\xi)} d \xi+e^{\theta(\eta) x}\{\hat{\phi}(\eta) \\
&\left.-\frac{1}{2 \theta(\eta)} \int_{0}^{\infty} e^{\theta(\eta) \xi} \frac{\hat{f}(\xi, \eta)}{\rho(\xi)} d \xi\right\} .
\end{aligned}
$$

Theorem 3.1. For $\hat{u}(x, \eta)$ given by $(3.8)$, we obtain

$$
\begin{gathered}
\int_{\mathbf{R}^{n-1}} \int_{0}^{\infty}\left(\left.\left.\rho(x) \sum_{\alpha_{1}+\alpha_{2}=2}\left|\left(\frac{d}{d x}\right)^{\alpha_{1}}\right| \eta\right|^{\alpha_{2}} \hat{u}(x, \eta)\right|^{2}+|\hat{u}(x, \eta)|^{2}\right) d x d \eta \leqq \\
\leqq C\left(\int_{\mathbf{R}^{n-1}}(1+|\eta|)^{3-\alpha}|\hat{\phi}(\eta)|^{2} d \eta+\int_{\mathbf{R}^{n-1}} \int_{0}^{\infty} \frac{|\hat{f}(x, \eta)|^{2}}{\rho(x)} d x d \eta\right) .
\end{gathered}
$$

Proof. Since $|\theta(\eta)| \leqq$ const. $-\operatorname{Re} \theta(\eta)$ holds by (3.6), we may assume $\theta(\eta)$ is real. First consider

$$
u_{1}(x, \eta)=e^{\theta(\eta) x}\left(\hat{\phi}(\eta)-\frac{1}{2 \theta(\eta)} \int_{0}^{\infty} e^{\theta(\eta) \xi} \frac{\hat{f}(\xi, \eta)}{\rho(\xi)} d \xi\right)
$$

Now we may assume $\rho(x) \leqq M x^{\alpha}$, we have

$$
\begin{aligned}
\int_{0}^{\infty} \rho(x)\left|\theta(\eta)^{2} e^{\theta(\eta) x} \hat{\phi}(\eta)\right|^{2} d x & \leqq M \theta(\eta)^{4}|\hat{\phi}(\eta)|^{2} \int_{0}^{\infty} x^{\alpha} e^{2 \theta(\eta) x} d x \\
& \leqq M \Gamma(1+\alpha)|\theta(\eta)|^{3-\alpha}|\hat{\phi}(\eta)|^{2}
\end{aligned}
$$

and this shows

$$
\begin{aligned}
& \int_{\mathbf{R}^{n-1}} \int_{0}^{\infty} \rho(x)\left|\theta(\eta)^{2} e^{\theta(\eta) x} \hat{\phi}(\eta)\right|^{2} d x d \eta \\
& \leqq M \Gamma(1+\alpha) \int_{\mathbf{R}^{n-1}}(1+|\eta|)^{3-\alpha}|\hat{\phi}(\eta)|^{2} d \eta
\end{aligned}
$$

Next set

$$
A(\eta)=\frac{1}{2 \theta(\eta)} \int_{0}^{\infty} e^{\theta(\eta) \xi} \frac{\hat{f}(\xi, \eta)}{\rho(\xi)} d \xi
$$


then we have

$$
\begin{aligned}
|\theta(\eta) A(\eta)|^{2} & \leqq \text { const. }\left(\int_{0}^{1} \frac{e^{2 \theta(\eta) \xi}}{\rho(\xi)} d \xi \int_{0}^{\infty} \frac{|\hat{f}(\xi, \eta)|^{2}}{\rho(\xi)} d \xi\right. \\
& \left.+\int_{0}^{\infty} e^{2 \theta(\eta) \xi} d \xi \int_{0}^{\infty} \frac{|\hat{f}(\xi, \eta)|^{2}}{\rho(\xi)} d \xi\right) \\
& \leqq \text { const. }|\theta(\eta)|^{\alpha-1} \int_{0}^{\infty} \frac{|\hat{f}(x, \eta)|^{2}}{\rho(x)} d x .
\end{aligned}
$$

Hence we have

$$
\begin{aligned}
\int_{0}^{\infty} \rho(x)\left|\theta(\eta)^{2} A(\eta)\right|^{2} e^{2 \theta(\eta) x} d x & \leqq \text { const. }|\theta(\eta)|^{1+\alpha} \int_{0}^{\infty} \rho(x) e^{2 \theta(\eta) x} d x \\
& \times \int_{0}^{\infty} \frac{|\hat{f}(x, \eta)|^{2}}{\rho(x)} d x \\
& \leqq \text { const. } \int_{0}^{\infty} \frac{|\hat{f}(x, \eta)|^{2}}{\rho(x)} d x,
\end{aligned}
$$

and then

(3.11) $\int_{\mathbf{R}^{n-1}} \int_{0}^{\infty} \rho(x)\left|\theta(\eta)^{2} A(\eta)\right|^{2} e^{2 \theta(\eta) x} d x d \eta \leqq$ const. $\int_{\mathbf{R}^{n-1}} \int_{0}^{\infty} \frac{|\hat{f}(x, \eta)|^{2}}{\rho(x)} d x d \eta$.

Together with (3.10) and (3.11), we obtain

$$
\begin{aligned}
& \int_{\mathbf{R}^{n-1}} \int_{0}^{\infty}\left(\left.\left.\rho(x) \sum_{\alpha_{1}+\alpha_{2}=2}\left|\left(\frac{d}{d x}\right)^{\alpha_{1}}\right| \eta\right|^{\alpha_{2}} u_{1}(x, \eta)\right|^{2}+\left|u_{1}(x, \eta)\right|^{2}\right) d x d \eta \leqq \\
& \leqq \text { const. }\left(\int_{\mathbf{R}^{n-1}}(1+|\eta|)^{3-\alpha}|\hat{\phi}(\eta)|^{2} d \eta+\int_{\mathbf{R}^{n-1}} \int_{0}^{\infty} \frac{|\hat{f}(x, \eta)|^{2}}{\rho(x)} d x d \eta\right) .
\end{aligned}
$$

Our next object is

$$
u_{2}(x, \eta)=\frac{1}{2 \theta(\eta)} \int_{0}^{\infty} e^{\theta(\eta)|x-\xi|} \frac{\hat{f}(\xi, \eta)}{\rho(\xi)} d \xi
$$

Now let $\beta_{1}(x)$ and $\beta_{2}(x)$ be the following functions;

$$
\beta_{1}(x)=0(0 \leqq x \leqq 1) \text { and }=1(1<x<\infty) \text {, }
$$




$$
\beta_{2}(x)=1(0 \leqq x \leqq 1) \text { and }=0 \quad(1<x<\infty)
$$

and put

$$
f_{1}(x, \eta)=\beta_{1}(x) f(x, \eta) \text { and } f_{2}(x, \eta)=\beta_{2}(x) f(x, \eta)
$$

then clearly

$$
f(x, \eta)=f_{1}(x, \eta)+f_{2}(x, \eta)
$$

Define now $v_{1}(x, \eta)$ and $v_{2}(x, \eta)$ by

$$
\begin{aligned}
& v_{1}(x, \eta)=\frac{1}{2 \theta(\eta)} \int_{0}^{\infty} e^{\theta(\eta)|x-\xi|} \frac{f_{1}(\xi, \eta)}{\rho(\xi)} d \xi \\
& v_{2}(x, \eta)=\frac{1}{2 \theta(\eta)} \int_{0}^{\infty} e^{\theta(\eta)|x-\xi|} \frac{f_{2}(\xi, \eta)}{\rho(\xi)} d \xi .
\end{aligned}
$$

For $v_{1}(x, \eta)$ by the Hausdorff-Young inequality we can easily show

$$
\int_{0}^{\infty} \theta(\eta)^{2}\left|v_{1}(x, \eta)\right|^{2} \rho(x) d x \leqq \sup _{x \geqq 1} \rho(x) \cdot \sup _{x \geqq 1} \frac{1}{\rho(x)} \int_{0}^{\infty} \frac{\left|f_{1}(x, \eta)\right|^{2}}{\rho(x)} d x
$$

and hence

(3.13) $\int_{\mathbf{R}^{n-1}} \int_{0}^{\infty} \rho(x)\left|\theta(\eta)^{2} v_{1}(x, \eta)\right|^{2} d x d \eta \leqq$ const. $\int_{\mathbf{R}^{n-1}} \int_{0}^{\infty} \frac{\left|f_{1}(x, \eta)\right|^{2}}{\rho(x)} d x d \eta$.

For $v_{2}(x, \eta)$, since we may assume $A_{1} x^{\alpha} \leqq \rho(x) \leqq A_{2} x^{\alpha}(0 \leqq x \leqq 1)$, it will be sufficient to consider

$$
v(x, \eta)=\frac{1}{2 \theta(\eta)} \int_{0}^{\infty} e^{\theta(\eta)|x-\xi|} \frac{f_{2}(\xi, \eta)}{\xi^{\alpha}} d \xi
$$

in place of $v_{2}(x, \eta)$. Our aim is to show

$$
\int_{0}^{\infty} x^{\alpha}\left|\theta(\eta)^{2} v(x, \eta)\right|^{2} d x \leqq \text { const. } \int_{0}^{\infty} \frac{\left|f_{2}(x, \eta)\right|^{2}}{x^{\alpha}} d x
$$

then (3.12) (3.13) and (3.14) will lead us to (3.9).

Since $f_{2}(x, \eta) x^{-\alpha / 2}$ belongs to $L_{x}^{2}(0, \infty)$, and since 


$$
2 x^{\alpha / 2} \theta(\eta)^{2} v(x, \eta)=\theta(\eta) \int_{0}^{\infty} e^{\theta(\eta)|x-\xi|}\left(\frac{x}{\xi}\right)^{\alpha / 2} \frac{f_{2}(\xi, \eta)}{\xi^{\alpha / 2}} d \xi
$$

by Proposition 3.1 we can obtain (3.14).

Though we investigated only tangential derivatives, the terms with cross derivatives, involving a first order normal derivative, are estimated as well as of tangential derivatives, and the term with second order normal derivative can be estimated from the equation (3.1).

Q.E.D.

Next consider the Neumann problem for special case;

$$
-\rho(x)\left(\frac{\partial^{2} u}{\partial x^{2}}+2 \sum_{j=1}^{n-1} a_{j} \frac{\partial^{2} u}{\partial x \partial y_{j}}+\sum_{j, k=1}^{n-1} b_{j k} \frac{\partial^{2} u}{\partial y_{j} \partial y_{k}}-\left(1+|\sigma|^{2}\right) u\right)=f(x, y)
$$

with boundary condition

$$
\frac{\partial u}{\partial x}+2 \sum_{j=1}^{n-1} a_{j} \frac{\partial u}{\partial y_{j}}+\left.\sigma u\right|_{x=0}=\phi
$$

where $a_{j}$ 's and $b_{j k}$ 's are as same as given in Theorem 3.1, and $\sigma$ is a constant number.

We assume $\phi(y)$ belongs to $H^{(1-\alpha) / 2}\left(\mathbf{R}^{n-1}\right)$ and

$$
\int_{\mathbf{R}^{n-1}} \int_{0}^{\infty} \frac{|f(x, y)|^{2}}{\rho(x)} d x d \eta<+\infty .
$$

Repeating the similar argument as for Dirichlet problem, we can prove the following theorem.

Theorem 3.2. If $u(x, y)$ belonging to $H^{2}\left(\mathbf{R}_{+}^{n}, \rho\right)$ satisfies (3.15) and (3.16), then it follows

$$
\begin{array}{r}
\int_{\mathbf{R}^{n-1}} \int_{0}^{\infty}\left(\left.\left.\rho(x) \sum_{\alpha_{1}+\alpha_{2}=2}\left|\left(\frac{d}{d x}\right)^{\alpha_{1}}\right| \eta\right|^{\alpha_{2}} u(x, \eta)\right|^{2}+|u(x, \eta)|^{2}\right) d x d \eta \leqq \\
\leqq C\left(\int_{\mathbf{R}^{n-1}}(1+|\eta|)^{1-\alpha}|\hat{\psi}(\eta)|^{2} d \eta+\int_{\mathbf{R}^{n-1}} \int_{0}^{\infty} \frac{|f(x, \eta)|^{2}}{\rho(x)} d x d \eta\right)
\end{array}
$$

where ^ denotes the tangential Fourier image. 


\section{Estimates for the General Cases}

The aim in this section is to establish a priori estimates for the solutions of (1.1) which belong to $H^{2}(\Omega, \rho)$, when $\Omega$ is a half-spaces or a general domain with smooth compact boundary.

The transition from cases with constant coefficients to cases with variable coefficients is routine, through the localization by the partition of unity.

Let $L^{\prime}\left(x, y ; D_{x}, D_{y}\right)$ be an elliptic operator defined in $\mathbf{R}_{+}^{n}$ :

$$
\begin{aligned}
L^{\prime}\left(x, y ; D_{x}, D_{y}\right)= & -\left(\frac{\partial}{\partial x} a_{00}(x, y) \frac{\partial}{\partial x}\right)+\sum_{j=1}^{n-1} \frac{\partial}{\partial x}\left(a_{0 j}(x, y) \frac{\partial}{\partial y_{j}}\right) \\
& +\frac{\partial}{\partial y_{j}}\left(a_{j 0}(x, y) \frac{\partial}{\partial x}\right)+\sum_{j, k=1}^{n-1} \frac{\partial}{\partial y_{j}}\left(a_{j k}(x, y) \frac{\partial}{\partial y_{k}}\right),
\end{aligned}
$$

where $a_{j k}(x, y)(j, k=0,1, \ldots, n-1) \in \mathscr{B}^{1}\left(\mathbf{R}_{+}^{n}\right)$ are all real valued symmetric and satisfy

$$
\sum_{j, k=0}^{n-1} a_{j k}(x, y) \xi_{j} \xi_{k} \geqq c|\xi|^{2} \quad(c>0) .
$$

We begin with the Lemma 4.1 below.

Let $u(x, y) \in H^{2}\left(\mathbf{R}_{+}^{n}, \rho\right)$ be a solution of

$$
\rho(x) L^{\prime}\left(x, y ; D_{x}, D_{y}\right) u=f(x, y)
$$

satisfying the boundary condition

$$
\left.u(x, y)\right|_{x=0}=\phi(y) \in H^{(3-\alpha) / 2}\left(\mathbf{R}^{n-1}\right)
$$

or

$$
\left.B\left(y ; D_{x}, D_{y}\right) u(x, y)\right|_{x=0}=\psi(y) \in H^{(1-\alpha) / 2}\left(\mathbf{R}^{n-1}\right),
$$

where $B\left(y ; D_{x}, D_{y}\right)$ is given by

$$
B\left(x ; D_{x}, D_{y}\right)=a_{00}(0, y) \frac{\partial}{\partial x}+\sum_{j=1}^{n-1} a_{0 j}(0, y) \frac{\partial}{\partial y_{j}}+\sigma(y)
$$


and $\sigma(y) \in \mathscr{B}^{1}\left(\mathbb{R}_{+}^{n}\right)$.

Lemma 4.1. For a solution $u \in H^{2}\left(\mathbf{R}_{+}^{n}, \rho\right)$ of (4.1), suppose that the support of $u(x, y)$ lies in a hemi-sphere $\Sigma_{\delta}$;

$$
\Sigma_{\delta}=\left\{(x, y) ; x^{2}+|y|^{2}<\delta^{2}, \text { and } x>0\right\}
$$

with sufficiently small $\delta>0$. We obtain

$$
\|u\|_{2, \rho} \leqq C\left(\|f\|_{0, \rho^{-1}}+|\phi|_{(3-\alpha) / 2}+\|u\|\right)
$$

when $u(x, y)$ satisfies (4.1), and obtain also

$$
\|u\|_{2, \rho} \leqq C\left(\|f\|_{0, \rho^{-1}}+|\psi|_{(1-\alpha) / 2}+\|u\|\right)
$$

when $u(x, y)$ satisfies (4.3). Here $\|f\|_{0, p^{-1}}$ denotes

$$
\|f\|_{0, \rho^{-1}}^{2}=\int_{\mathbf{R}_{+}^{n}} \frac{|f(x, y)|^{2}}{\rho(x)} d x d y .
$$

Proof. (I) The case when $u(x, y)$ satisfies (4.2).

Rewrite (4.1) as

$$
\begin{aligned}
\rho(x) L^{\prime}\left(0,0 ; D_{x}, D_{y}\right) u= & f(x, y)-\rho(x)\left(L^{\prime}\left(x, y ; D_{x}, D_{y}\right)\right. \\
& \left.-L^{\prime}\left(0,0 ; D_{x}, D_{y}\right)\right) u \\
\equiv & g(x, y) .
\end{aligned}
$$

Of course $\|g\|_{0, \rho^{-1}}<+\infty$, and using Theorem 3.1, we obtain

$$
\|u\|_{2, \rho} \leqq \text { const. }\left(\|g\|_{0, \rho^{-1}}+\|u\|+|\phi|_{(3-\alpha) / 2}\right) \text {. }
$$

Hence by an obvious estimate

$$
\|g\|_{0, \rho^{-1}} \leqq\|f\|_{0, \rho^{-1}}+\left\|\sqrt{\rho(x)}\left(L^{\prime}\left(x, y ; D_{x}, D_{y}\right) u-L^{\prime}\left(0,0 ; D_{x}, D_{y}\right) u\right)\right\|,
$$

and by the continuity and the boundedness of first derivatives of the coefficients, we have

$$
\|u\|_{2, \rho} \leqq C\left(\left(\|f\|_{0, \rho^{-1}}+|\phi|_{(3-\alpha) / 2}\right)+\varepsilon\|u\|_{2, \rho}+C^{\prime}\|u\|_{1}\right),
$$


where $\varepsilon \rightarrow 0$ as $\delta \rightarrow 0$. Thus taking $\delta$ sufficiently small and noticing Proposition 2.1, we can obtain (4.4) easily.

(II) The case when $u(x, y)$ satisfies (4.3).

Rewrite (4.3) as

$\left.B\left(0 ; D_{x}, D_{y}\right) u(x, y)\right|_{x=0}=\psi(y)+\left.\left\{B\left(0 ; D_{x}, D_{y}\right)-B\left(y ; D_{x}, D_{y}\right) u(x, y)\right\}\right|_{x=0}$.

Using (4.6) and applying Theorem 3.2, we have

$$
\begin{aligned}
\|u\|_{2, \rho} \leqq C\left(\|f\|_{0, \rho^{-1}}\right. & \left.+|\psi|_{(1-\alpha) / 2}\right)+\varepsilon\|u\|_{2, \rho}+C^{\prime}\|u\|_{1}+ \\
& +\left|\left(B\left(y ; D_{x} ; D_{y}\right) u-B\left(0 ; D_{x}, D_{y}\right) u\right)\right|_{(1-\alpha) / 2}
\end{aligned}
$$

On the other hand, according to Theorem 2.1, it follows

$$
\begin{aligned}
& \left|\left(B\left(y ; D_{x}, D_{y}\right) u-B\left(0 ; D_{x}, D_{y}\right) u\right)\right|_{(1-\alpha) / 2} \leqq \\
& \leqq C_{\|}\left\|\left(B\left(y ; D_{x}, D_{y}\right)-B\left(0 ; D_{x}, D_{y}\right)\right) u\right\|_{1, \rho},
\end{aligned}
$$

and hence

$$
\leqq \varepsilon^{\prime}\|u\|_{2, \rho}+C^{\prime \prime}\|u\|_{1, \rho},
$$

where $\varepsilon^{\prime} \rightarrow 0$ as $\delta \rightarrow 0$ (because of the continuity of the coefficients). Thus we can obtain (4.5) as well as (4.4).

Q.E.D.

Next consider the case when the support of $u(x, y)$ extends over $\mathbf{R}_{+}^{n}$. The following proposition follows from Lemma 4.1.

Proposition 4.1. Suppose that $u(x, y) \in H^{2}\left(\mathbf{R}_{+}^{n}, \rho\right)$ satisfies (4.1) and (4.2) or (4.3). It follows

$$
\|u\|_{2, \rho} \leqq C\left(\|f\|_{0, \rho^{-1}}+|\phi|_{(3-\alpha) / 2}+\|u\|\right)
$$

when $u(x, y)$ satisfies (4.2), and

$$
\|u\|_{2, \rho} \leqq C\left(\|f\|_{0, \rho^{-1}}+|\psi|_{(1-\alpha) / 2}+\|u\|\right)
$$

when $u(x, y)$ satisfies (4.3).

Proof. Let us notice that we can take a locally finite partition of 
unity $\left\{\omega_{p}(x, y)\right\}$ of $\mathbf{R}_{+}^{n}$ as follows;

(1) $\sum \omega_{p}(x, y)=1$ and $0 \leqq \omega_{p}(x, y) \leqq 1$

(2) inf diam. of support $\omega_{p}(x, y)=d>0$

(3) the number of the supports which intersect mutually is limited by a suitable integer $N$

(4) each of the diameter of support of $\omega_{p}(x, y)$ sufficiently small such as Lemma 4.1 is applicable.

Localizing (4.1) and (4.2) or (4.3) by the above partition of unity, and applying Lemma 4.1, we can establish our assertion. Q.E.D.

To end this section let us consider the case of general domain $\Omega$. Since the interior estimates are well known and the essential parts for the estimates near the boundary were carried out in Lemma 4.1, we state only results.

Theorem 4.1. Let $\Omega$ be a domain in $\mathbb{R}^{n}$ whose boundary is a smooth compact hypersurface, then, if $u(x)$ belonging to $H^{2}(\Omega, \rho)$ satisfies (1.1) with $f(x) \in L^{2}\left(\Omega, \rho^{-1}\right)$ and satisfies the boundary condition (1.4) or (1.5), it holds

$$
\|u\|_{2, \rho} \leqq C\left(\|f\|_{0, \rho^{-1}}+|\phi|_{(3-\alpha) / 2}+\|\left. u\right|_{i}\right)
$$

or

$$
\|u\|_{2, \rho} \leqq C\left(\|f\|_{0, \rho^{-1}}+|\psi|_{(1-\alpha) / 2}+\|u\|\right)
$$

corresponding to each boundary condition.

\section{Weak Solutions}

As for the existence of solution, it will be essentially based on the well-known Riesz' representation theorem. In this section we define the weak solution of (homogeneous) Dirichlet problem and (homogeneous) Neumann problem, and we show there exists a unique weak solution.

Definition 5.1. $u(x) \in \mathscr{D}_{L^{2}}^{1}(\Omega)$ is called a weak solution of (1.1) with boundary condition 


$$
\left.u(x)\right|_{\Gamma}=0
$$

if and only if $u(x)$ satisfies

$$
\begin{aligned}
D[u, v]= & \sum_{j, k=1}^{n}\left(a_{j k}(x) \frac{\partial u}{\partial x_{k}}, \frac{\partial v}{\partial x_{j}}\right)+\left(\sum_{j=1}^{n}\left(b_{j}(x) \frac{\partial u}{\partial x_{j}}, v\right)+(c(x) u, v)+\right. \\
& \sum_{j=1}^{n}\left(p_{j}(x) \frac{\partial u}{\partial x_{j}}, v\right)_{\rho^{-1}}+(q(x) u, v)_{\rho^{-1}}=(f, v)_{\rho^{-1}}
\end{aligned}
$$

for all $v(x) \in \mathscr{D}_{L^{2}}^{1}(\Omega)$, where $($,$) denotes the inner product of L^{2}(\Omega)$ and $(,)_{\rho^{-1}}$ the inner product of $L^{2}\left(\Omega, \rho^{-1}\right)$.

The well-definedness of (5.1) follows from

Lemma 5.1. For any $u(x)$ and $v(x)$ in $\mathscr{D}_{L^{2}}(\Omega)$, it follows

$$
\left|(D u, v)_{p^{-1}}\right| \leqq\|u\|_{1}\left(\varepsilon\|v\|_{1}+C(\varepsilon)\|v\|\right)
$$

where $D$ is an arbitrary first order differential operator with bounded coefficients and $\varepsilon$ is an arbitrary positive number.

And

Lemma 5.2. If $f(x)$ is in $L^{2}\left(\Omega, \rho^{-1}\right)$ and $v(x)$ in $H^{1}(\Omega)$, then it follows

$$
\left|(f, v)_{\rho^{-1}}\right| \leqq\|f\|_{0, \rho^{-1}}\left(\varepsilon\|v\|_{1}+C_{\varepsilon}\|v\|\right)
$$

for any $\varepsilon>0$.

Proof of Lemma 5.1. It will be sufficient to prove when $\Omega$ is $\mathbf{R}_{+}^{n}$ and $\rho$ depends only on normal variable $x$.

Since $v(x, y)$ belongs to $\mathscr{D}_{L^{2}}^{1}\left(\mathbf{R}_{+}^{n}\right)$, we have for almost every $y$

$$
|v(x, y)|^{2} \leqq x \int_{0}^{\infty}\left|\frac{\partial u}{\partial x}\right|^{2} d x,
$$

and hence for any $\delta>0$ we get

$$
\int_{0}^{\delta} \frac{|v(x, y)|^{2}}{\rho(x)^{2}} d x \leqq \text { const. } \delta^{2-2 \alpha} \int_{0}^{\infty}\left|\frac{\partial u}{\partial x}\right|^{2} d x .
$$


On the other hand, we have

$$
\int_{\delta}^{\infty} \frac{|v(x, y)|^{2}}{\rho(x)} d x \leqq \sup _{x \geqslant \delta} \frac{1}{\rho(x)^{2}} \int_{0}^{\infty}|v(x, y)|^{2} d x .
$$

Thus we obtain

$$
\int_{\mathbf{R}_{+}^{n}} \frac{|v(x, y)|^{2}}{\rho(x)^{2}} d x d y \leqq \text { const. } \delta^{2-2 \alpha}\|v\|_{1}^{2}+C(\delta)\|v\|^{2}
$$

and using Schwarz' inequality we can have (5.2) easily.

Q.E.D.

Proof of Lemma 5.2. By similar technique used in the proof of Lemma 5.1 , we can obtain for any $v(x)$ in $H^{1}\left(\mathbb{R}_{+}^{n}\right)$

$$
\int_{\mathbf{R}_{+}^{n}} \frac{|v(x, y)|^{2}}{\rho(x)} d x d y \leqq \varepsilon\|v\|_{1}^{2}+C(\varepsilon)\|v\|^{2},
$$

then this and Schwarz' inequality yield (5.3).

As a corollary of Lemma 5.2 , we have

Lemma 5.3. If $u(x)$ belongs to $\mathscr{D}_{L^{2}}(\Omega)$, then it follows

$$
\left|(D u, u)_{\rho^{-1}}\right| \leqq \varepsilon\|u\|_{1}^{2}+C(\varepsilon)\|u\|^{2},
$$

for any $\varepsilon>0$, here $D$ is as same as given in Lemma 5.1 .

The following theorem stating the existence of weak solution of homogeneous Dirichlet problem is an immediate consequence of Lemma 5.2, Lemma 5.3 and the well-known Riesz' representation theorem or Lax-Milgram lemma.

Theorem 5.1. For any $f(x)$ in $L^{2}\left(\Omega, \rho^{-1}\right)$, there exists a real number $\lambda_{0}$ such that for any $\lambda \geqq \lambda_{0}$ the equation

$$
\left\{\begin{array}{c}
\rho(x) L(x, D) u+\sum_{j=1}^{n} p_{j}(x) \frac{\partial u}{\partial x_{j}}+q(x) u+\lambda u=f(x) \\
\left.u\right|_{\Gamma}=0
\end{array}\right.
$$


has a unique weak solution.

Proof. It will suffices to notice that it follows

(1) $\left|D_{\lambda}[u, v]\right| \leqq C\left\|_{\mid} u\right\|_{1}\|v\|_{1}$

(2) $\operatorname{Re} D_{\lambda}[u, u] \geqq C\|u\|_{1}^{2}$

for any $u(x)$ and $v(x)$ in $\mathscr{D}_{L^{2}}(\Omega)$, where $D_{\lambda}[u, v]=D[u, v]+\lambda(u, v)_{\rho^{-1}}$, and that $(f, \cdot)$ gives a linear bounded functional on $\mathscr{D}_{L^{2}}^{1}(\Omega)$ when $f(x)$ belongs to $L^{2}\left(\Omega, \rho^{-1}\right)$.

Q.E.D.

Now let us define a weak solution of Neumann problem with homogeneous boundary condition. For general equation it is difficult to define the weak solution with good nature, so we define it for rather special equation, more precisely we assume here

$$
p_{j}(x) \equiv 0 \quad(j=1,2, \ldots, n) .
$$

Definition 5.2. $u(x)$ in $H^{1}(\Omega)$ is said to be a weak solution of the equation

$$
\begin{gathered}
\rho(x) L(x, D) u+q(x) u=f(x) \\
\frac{\partial u}{\partial \nu}+\left.\sigma u\right|_{\Gamma}=0,
\end{gathered}
$$

if and only if $u(x)$ satisfies

$$
\begin{array}{r}
N[u, v] \equiv \sum_{j, k=1}^{n}\left(a_{j k}(x) \frac{\partial u}{\partial x_{k}}, \frac{\partial v}{\partial x_{j}}\right)+\sum_{j=1}^{n}\left(b_{j}(x) \frac{\partial u}{\partial x_{j}}, v\right)+(c(x) u, v)+ \\
+(q(x) u, v)_{\rho^{-1}}+\left\langle\sigma u, v>_{\Gamma}=(f, v)\right.
\end{array}
$$

for all $v(x)$ in $H^{1}(\Omega)$, where $<,>_{\Gamma}$ denotes the inner product of $L_{2}(\Gamma)$.

Well-definedness of $N[u, v]$ follows from

Lemma 5.4. If $u(x)$ is in $H^{1}(\Omega)$, then it holds

$$
\|u\|_{0, \rho^{-1}} \leqq \varepsilon\|u\|_{1}+C(\varepsilon)\|u\|
$$

for any $\varepsilon>0$. 
Proof. It will be sufficient to show (5.7) when $\Omega=\mathbf{R}_{+}^{n}$ and $\rho$ depends only on the normal variable $x$.

Noticing that

$$
u(x, y)=u(0, y)+\int_{0}^{x} \frac{\partial u}{\partial \xi} d \xi
$$

we have

$$
|u(x, y)|^{2} \leqq 2\left(|u(0, y)|^{2}+x \int_{0}^{\infty}\left|\frac{\partial u}{\partial x}\right|^{2} d x\right)
$$

hence for any $\delta>0$

$$
\int_{0}^{\delta} \frac{|u(x, y)|^{2}}{\rho(x)} d x \leqq K(\delta)\left(|u(0, y)|^{2}+\int_{0}^{\infty}\left|\frac{\partial u}{\partial x}\right|^{2} d x\right)
$$

where $K(\delta) \rightarrow 0$ when $\delta \rightarrow 0$.

On the other hand we have

$$
\int_{\delta}^{\infty} \frac{|u(x, y)|^{2}}{\rho(x)} d x \leqq \sup _{x \geqq \delta} \frac{1}{\rho(x)} \int_{0}^{\infty}|u(x, y)|^{2} d x
$$

Thus we obtain

$$
\left.\int_{\mathbf{R}_{+}^{n}} \frac{|u(x, y)|^{2}}{\rho(x)} d x d y \leqq K(\delta)\left(\|u\|_{1}^{2}+\int_{\mathbf{R}^{n-1}}|u(0, y)|^{2} d y\right)+\sup _{x \geqq \delta} \frac{1}{\rho(x)}\|u\|^{2}\right)
$$

and taking $\delta$ sufficiently small, we can obtain (5.7).

Q.E.D.

The existence of the weak solution can be established through the same manner as the case of Dirichlet problem.

Theorem 5.2. For any $f(x)$ in $L^{2}\left(\Omega, \rho^{-1}\right)$, there exists a real number $\lambda_{0}$ such that for any $\lambda \geqq \lambda_{0}$ the equation

$$
\begin{gathered}
\rho(x) L(x, D) u+q(x) u+\lambda u=f(x) \\
\frac{\partial u}{\partial \nu}+\left.\sigma u\right|_{r}=0
\end{gathered}
$$


has a unique weak solution.

\section{Proof of Theorem $\mathbb{1} .1$ (I)}

Now that the existence of weak solution has been established for each equation, our main aim is to show the regularity of weak solution, more precisely to show that the weak solution obtained in the previous section belongs to $H^{2}(\Omega, \rho)$ in each case, then it will prove Theorem 1.1. In this section we shall give a proof of Theorem 1.1 for special equation, where $p_{j}(x) \equiv 0$ in (1.1), that is to say,

$$
\rho(x) L(x, D) u+q(x) u+\lambda u=f(x)
$$

with homogeneous Dirichlet or Neumann condition.

The main tool to our aim is the tangential mollifier of Friedrichs, so we describe a few lemmas of mollifier.

Let us denote by $\varphi_{\varepsilon} *$ the tangential mollifier:

$$
\varphi_{\varepsilon} * u(x, y)=\int_{\mathbf{R}^{-1}} \varphi_{\varepsilon}(y-\eta) u(x, \eta) d \eta
$$

where $\varphi_{\varepsilon}(y)=\varepsilon^{1-n} \varphi(y / \varepsilon)$ with $\varphi \in C_{0}^{\infty}\left(\mathbf{R}^{n-1}\right)$ such that $\int \varphi(y) d y=1$.

We shall write $u_{\varepsilon}(x, y)=\varphi_{\varepsilon} * u(x, y)$ in what follows.

Lemma 6.1. If $u(x, y)$ belongs to $L^{2}\left(\mathbf{R}_{+}^{n}, \rho^{-1}\right),{ }^{1)}$ then $u_{\varepsilon}(x, y)$ goes to $u(x, y)$ in $L^{2}\left(\mathbb{R}_{+}^{n}, \rho^{-1}\right)$ as $\varepsilon \rightarrow 0$.

Lemma 6.2. (Friedrichs) Let $u(x, y)$ be in $H^{1}\left(\mathbb{R}_{+}^{n}\right)$ and $a(x, y)$ be in $\mathscr{B}^{1}\left(\mathbf{R}_{+}^{n}\right)$, then $\left[\varphi_{\varepsilon} *, a(x, y) D\right] u$ converges to 0 in $H^{1}\left(\mathbf{R}_{+}^{n}\right)$ as $\varepsilon \rightarrow 0$, where $D$ stands for $\partial / \partial x$ or $\partial / \partial y_{j}(j=1, \ldots, n-1)$.

Lemma 6.3. Let $v(y)$ be in $H^{1 / 2}\left(\mathbb{R}^{n-1}\right)$ and $b(y)$ be in $\mathscr{B}^{1}\left(\mathbb{R}^{n-1}\right)$, then $\left[\varphi_{\varepsilon} *, b(y)\right] v$ converges to 0 in $H^{1 / 2}\left(\mathbb{R}^{n-1}\right)$ as $\varepsilon \rightarrow 0$.

Now back to show the regularity of our weak solutions. Since the interior regularity is well known, we shall show only the regularity near the boundary. Noticing that the problem is completely local, it suffices to

1) When we use the notation $L^{2}\left(\mathbf{R}_{+}^{n}, \rho^{-1}\right)$, we assume $\rho$ depends only on $x$. 
show it when $\Omega$ is a half-space after a local transformation of independent variables. Let our concerning equation be

$$
\begin{aligned}
& -\rho(x)\left(\frac{\partial}{\partial x}\left(a_{00} \frac{\partial u}{\partial x}\right)+\sum_{j=1}^{n-1}\left(\frac{\partial}{\partial x}\left(a_{0 j} \frac{\partial u}{\partial y_{j}}\right)+\frac{\partial}{\partial y_{j}}\left(a_{0 j} \frac{\partial u}{\partial x}\right)\right)\right. \\
& \left.+\sum_{j, k=1}^{n-1} \frac{\partial}{\partial y_{j}}\left(a_{j k} \frac{\partial u}{\partial y_{k}}\right)+b_{0} \frac{\partial u}{\partial x}+\sum_{j=1}^{n-1} b_{j} \frac{\partial u}{\partial y_{j}}+c u\right)+q u+\lambda u=f(x, y)
\end{aligned}
$$

and

$$
\left.u(x, y)\right|_{x=0}=0
$$

or

$$
a_{00}(x, y) \frac{\partial u}{\partial x}+\sum_{j=1}^{n-1} a_{0 j}(x, y) \frac{\partial u}{\partial y_{j}}-\left.\sigma(y) u\right|_{x=0}=0
$$

where $a_{j k}(x, y)(j, k=0,1, \ldots, n-1) \in \mathscr{B}^{1}\left(\mathbb{R}_{+}^{n}\right)$, and $b_{j}(x, y)(j=0,1, \ldots$, $n-1), c(x, y)$ and $q(x, y)$ are all bounded and measurable, and $\sigma(y) \in$ $\mathscr{B}^{1}\left(\mathbb{R}^{n-1}\right)$.

Now since our weak solution belongs to $H^{1}\left(\mathbf{R}_{+}^{n}\right)$ and $H^{1}\left(\mathbb{R}_{+}^{n}\right)$ can be imbedded continuously into $L^{2}\left(\mathbf{R}_{+}^{n}, \rho^{-1}\right.$ ) (see the proof of Lemma 5.2), we may neglect the lower order term

$$
-\rho(x)\left(b_{0} \frac{\partial u}{\partial x}+\sum_{j=1}^{n-1} b_{j} \frac{\partial u}{\partial y_{j}}+c u\right)+q u+\lambda u
$$

by transferring it to the right hand side, and we treat the following equation

$$
\begin{aligned}
& -\rho(x)\left(\frac{\partial}{\partial x}\left(a_{00} \frac{\partial u}{\partial x}\right)+\sum_{j=1}^{n=1}\left(\frac{\partial}{\partial x}\left(a_{0 j} \frac{\partial u}{\partial y_{j}}\right)+\frac{\partial}{\partial y_{j}}\left(a_{j 0} \frac{\partial u}{\partial x}\right)\right)\right. \\
& \left.+\sum_{j, k=1}^{n=1} \frac{\partial}{\partial y_{j}}\left(a_{j k} \frac{\partial u}{\partial y_{k}}\right)\right)=f(x, y),
\end{aligned}
$$

in place of (6.1). 
Theorem 6.1. Let $u(x, y)$ be a weak solution of (6.4) with satisfying (6.2) or (6.3), then $u(x, y)$ belongs to $H^{2}\left(\mathbf{R}_{+}^{n}, \rho\right)$, where $f(x, y)$ is in $L^{2}\left(\mathbf{R}_{+}^{n}, \rho^{-1}\right)$ and $\rho$ depends only on $x$.

Proof. Consider the case of Neumann problem, because the Dirichlet case will be slightly easier, though the essential part is the same.

Now let $u(x, y)$ be a weak solution of (6.4) with boundary condition (6.3), then $u(x, y)$ satisfies for all $v(x, y)$ in $H^{1}\left(\mathbf{R}_{+}^{n}\right)$

$$
\begin{aligned}
& \text { (6.5) }\left(a_{00} \frac{\partial u}{\partial x}, \frac{\partial v}{\partial x}\right)+\sum_{j=1}^{n-1}\left(a_{0 j} \frac{\partial u}{\partial y_{j}}, \frac{\partial v}{\partial x}\right)+\sum_{j=1}^{n-1}\left(a_{j 0} \frac{\partial u}{\partial x}, \frac{\partial v}{\partial y_{j}}\right)+\sum_{j, k=1}^{n-1}\left(a_{j k} \frac{\partial u}{\partial y_{k}}, \frac{\partial u}{\partial y_{j}}\right) \\
& -\int_{\mathbf{R}^{n-1}} \sigma(y) u(0, y) \overline{v(0, y)} d y=(f, v)_{\rho^{-1}},
\end{aligned}
$$

and satisfies (6.4) as a distribution. Put here $v(x, y)=w(x, y) / a_{00}(x, y)$ with $w(x, y) \in H^{1}\left(\mathbf{R}_{+}^{n}\right)$ (notice here $a_{00}(x, y) \geqq c>0$ by uniform ellipticity), then (6.5) turns into

$$
\begin{gathered}
\left(\frac{\partial u}{\partial x}, \frac{\partial w}{\partial x}\right)+\sum_{j=1}^{n-1}\left(A_{0 j} \frac{\partial u}{\partial y_{j}}, \frac{\partial w}{\partial x}\right)+\sum_{j=1}^{n-1}\left(A_{j 0} \frac{\partial u}{\partial x}, \frac{\partial w}{\partial y_{j}}\right)+\sum_{j, k=1}^{n-1}\left(A_{j k} \frac{\partial u}{\partial y_{k}}, \frac{\partial u}{\partial y_{j}}\right)+ \\
+\left(\frac{\partial a}{\partial x} a_{00} \frac{\partial u}{\partial x}+\sum_{j=1}^{n-1} \frac{\partial a}{\partial x} a_{0 j} \frac{\partial u}{\partial y_{j}}+\sum_{j=1}^{n-1} \frac{\partial a}{\partial y_{j}} a_{j 0} \frac{\partial u}{\partial x}+\sum_{j, k=1}^{n-1} \frac{\partial a}{\partial y_{j}} a_{j k} \frac{\partial u}{\partial y_{k}}, w\right) \\
-\int_{\mathbf{R}^{n-1}} a(0, y) \sigma(y) u(0, y) \overline{w(0, y)} d y=(F, w)_{\rho^{-1}},
\end{gathered}
$$

where $A_{j k}(x, y)=a_{j k}(x, y) / a_{00}(x, y)(j, k=0,1, \ldots, n-1), F(x, y)=$ $f(x, y) / a_{00}(x, y)$ and $a(x, y)=1 / a_{00}(x, y)$. Hence, we may assume $a_{00}(x, y)=1$ identically in (6.5), since the second line of (6.6) can be transferred to the right hand side. We are now going to consider, instead of (6.5), the following

$$
\begin{gathered}
\left(\frac{\partial u}{\partial x}, \frac{\partial v}{\partial x}\right)+\sum_{j=1}^{n-1}\left(\left(a_{0 j} \frac{\partial u}{\partial y_{j}}, \frac{\partial v}{\partial x}\right)+\left(a_{j 0} \frac{\partial u}{\partial x}, \frac{\partial v}{\partial x_{j}}\right)\right)+\sum_{j, k=1}^{n-1}\left(a_{j k} \frac{\partial u}{\partial y_{k}}, \frac{\partial u}{\partial y_{j}}\right)- \\
-\int_{\mathbf{R}^{n-1}} \sigma(y) u(0, y) \overline{v(0, y)} d y=(f, v)_{\rho^{-1}}
\end{gathered}
$$

Now insert $v_{\varepsilon}$ in place of $v$ in (6.7), then we have by simple calculus 


$$
\begin{aligned}
& \left(\frac{\partial u_{\varepsilon}}{\partial x}, \frac{\partial v}{\partial x}\right)+\sum_{j=1}^{n-1}\left(\left(a_{0 j} \frac{\partial u_{\varepsilon}}{\partial y_{j}}, \frac{\partial v}{\partial x}\right)+\left(a_{j 0} \frac{\partial u_{\varepsilon}}{\partial x}, \frac{\partial v}{\partial y_{j}}\right)\right)+\sum_{j, k=1}^{n-1}\left(a_{j k} \frac{\partial u_{\varepsilon}}{\partial y_{k}}, \frac{\partial v}{\partial y_{j}}\right)- \\
- & \int_{\mathbf{R}^{n-1}} \sigma(y) u_{\varepsilon}(0, y) \overline{v(0, y)} d y+\sum_{j=1}^{n-1}\left(\left[\varphi_{\varepsilon} *, a_{j 0} \frac{\partial}{\partial x}\right] u, \frac{\partial v}{\partial y_{j}}\right)+ \\
+ & \sum_{j=1}^{n-1}\left(\left[\varphi_{\varepsilon} *, a_{0 j} \frac{\partial}{\partial y_{j}}\right] u, \frac{\partial v}{\partial x}\right)+\sum_{j, k=1}^{n-1}\left(\left[\varphi_{\varepsilon} *, a_{j k} \frac{\partial}{\partial y_{k}}\right] u, \frac{\partial v}{\partial y_{j}}\right)+ \\
- & \int_{\mathbf{R}^{n-1}}\left\{\left[\varphi_{\varepsilon} *, \sigma(y)\right] u(0, y)\right\} \overline{v(0, y)} d y=\left(f_{\varepsilon}, v\right)_{\rho^{-1}},
\end{aligned}
$$

and this shows that $u_{\varepsilon}(x, y)$ satisfies

$$
\begin{aligned}
& -\rho(x)\left(\frac{\partial^{2} u_{\varepsilon}}{\partial x^{2}}+\sum_{j=1}^{n-1}\left(\frac{\partial}{\partial x}\left(a_{0 j} \frac{\partial u_{\varepsilon}}{\partial y_{j}}\right)+\frac{\partial}{\partial y_{j}}\left(a_{j 0} \frac{\partial u_{\varepsilon}}{\partial x}\right)\right)+\sum_{j, k=1}^{n-1} \frac{\partial}{\partial y_{j}}\left(a_{j k} \frac{\partial u_{\varepsilon}}{\partial y_{k}}\right)\right)- \\
& -\rho(x)\left(\sum_{j=1}^{n-1}\left(\frac{\partial}{\partial x}\left(\left[\varphi_{\varepsilon} *, a_{0 j} \frac{\partial}{\partial y_{j}}\right] u\right)+\frac{\partial}{\partial y_{j}}\left(\left[\varphi_{\varepsilon} *, a_{j 0} \frac{\partial}{\partial x}\right] u\right)\right)+\right. \\
& \left.+\sum_{j, k=1}^{n-1} \frac{\partial}{\partial y_{j}}\left(\left[\varphi_{\varepsilon} *, a_{j k} \frac{\partial}{\partial y_{k}}\right] u\right)\right)=f_{\varepsilon}(x, y)
\end{aligned}
$$

in the sense of distribution. On the other hand, since $u(x, y)$ belongs to $H^{1}\left(\mathbf{R}_{+}^{n}\right)$, the terms involving the commutators in (6.9) belongs to $L^{2}\left(\mathbf{R}_{+}^{n}\right.$, $\rho^{-1}$ ) (cf. Lemma 6.2), and since $u_{\varepsilon}(x, y)$ is smooth in $y$, we can see that $u_{\varepsilon}(x, y)$ belongs to $H^{2}\left(\mathbb{R}_{+}^{n}, \rho\right)$ from the equation (6.9). Hence using the integration by parts, we can see that $u_{\varepsilon}(x, y)$ satisfies (6.9) in $L^{2}\left(\mathbf{R}_{+}^{n}, \rho^{-1}\right)$ and

$$
\begin{aligned}
& \frac{\partial u_{\varepsilon}}{\partial x}+\sum_{j=1}^{n-1} a_{0 j}(x, y) \frac{\partial u_{\varepsilon}}{\partial y_{j}}-\left.\sigma(y) u_{\varepsilon}\right|_{x=0}=\left.\left[\varphi_{\varepsilon} *, \sigma(y)\right] u\right|_{x=0} \\
& \quad-\left.\sum_{j=1}^{n-1}\left[\varphi_{\varepsilon} *, a_{0 j} \frac{\partial}{\partial y_{j}}\right] u\right|_{x=0} \cdot
\end{aligned}
$$

Thus applying Proposition 4.1 (a priori estimate) and noticing Lemma 6.1, Lemma 6.2 and Lemma 6.3 , we can see that $u_{\varepsilon}(x, y)$ converges in $H^{2}\left(\mathbf{R}_{+}^{n}, \rho\right)$ as $\varepsilon$ tends to 0 . On the other hand $u_{\varepsilon}(x, y)$ tends to $u(x, y)$ in $H^{1}\left(\mathbb{R}_{+}^{n}\right)$ clearly. Hence $u(x, y)$ has to lie in $H^{2}\left(\mathbf{R}_{+}^{n}, \rho\right)$ and this establishes 
the Theorem 1.1 in the case where $p_{j}(x) \equiv 0$.

Q.E.D.

\section{Proof of Theorem 1.1 (II)}

In this section, we shall finish the proof of the Theorem 1.1 by treating the general case where $p_{j}(x) \not 0$ in (1.1). We shall carry out this by a successive approximation with the aid of Proposition 4.1, Theorem 6.1 and the following

Lemma 7.1. Let $u(x)$ be a solution in $H^{2}(\Omega, \rho)$ of

$$
-\rho(x) L(x, D) u+q(x) u+\lambda u=f(x)
$$

satisfying the homogeneous Dirichlet or Neumann condition, then if $\lambda$ is sufficiently large, we obtain

$$
\|u\|_{0, p^{-1}} \leqq 1 /(\lambda-c)\|f\|_{0, \rho^{-1}}
$$

with some constant $c(<\lambda)$.

Proof of Theorem 1.1. According to Theorem 2.2 and Theorem 2.3 , it is sufficient to consider only the case of homogeneous boundary condition. But the Dirichlet case is already finished by Lemma 5.1 , therefore we restrict ourselves to the condition (4.3) (with $\psi=0$ ).

Now let us define $u_{k}(x)$ successively by

$$
-\rho(x) L(x, D) u_{k}+P(x, D) u_{k-1}+q(x) u_{k}+\lambda u_{k}=f(x)\left(u_{0}=0\right)
$$

with satisfying homogeneous boundary condition, where $P(x, D)=\sum_{j=1}^{n} p_{j}(x)$ $\frac{\partial}{\partial x_{j}}$ and is assumed sufficiently large so that (7.2) has a weak solution for $k=1$.

Let us note, by Theorem 6.1 , that each $u_{k}(x)$ lies in $H^{2}(\Omega, \rho)$, and hence our aim is to show that $u_{k}(x)$ converges in $H^{2}(\Omega, \rho)$ if we take $\lambda$ sufficiently large.

Now set $v_{k}(x)=u_{k}(x)-u_{k-1}(x)$, then $v_{k}(x)$ satisfies

$$
\begin{array}{r}
-\rho(x) L(x, D) v_{k}+q(x) v_{k}+\lambda v_{k}=-P(x, D) v_{k-1} \\
\quad(k=2,3, \ldots)
\end{array}
$$


and homogeneous boundary condition. Hence applying Theorem 4.1 to (7.3), and noticing Lemma 7.1, we obtain

$$
\left\|v_{k}\right\|_{2, \rho} \leqq C\left\|P(x, D) v_{k-1}\right\|_{0, \rho^{-1}} \quad(k=2,3, \ldots),
$$

where $C=C(\lambda)$ is uniformly bounded in $\lambda$. On the other hand owing to Proposition 2.1, we have

$$
\left\|v_{k}\right\|_{2, \rho} \leqq \varepsilon\left\|v_{k-1}\right\|_{2, \rho}+C(\varepsilon)\left\|v_{k-1}\right\|_{0, \rho^{-1}}
$$

and

$$
\left\|v_{k}\right\|_{0, \rho^{-1}} \leqq \frac{1}{\lambda-c}\left(\varepsilon\left\|v_{k-1}\right\|_{2, \rho}+C(\varepsilon)\left\|v_{k-1}\right\|_{0, \rho^{-1}}\right) .
$$

Now multiplying $C(\varepsilon) / \varepsilon$ to both sides of (7.6) and then adding it to (7.5), we have

(7.7) $\left\|v_{k}\right\|_{2, \rho}+\frac{C(\varepsilon)}{\varepsilon}\left\|v_{k}\right\|_{0, \rho^{-1}} \leqq\left(\varepsilon+\frac{C(\varepsilon)}{\lambda-c}\right)\left(\left\|v_{k-1}\right\|_{2, \rho}+\frac{C(\varepsilon)}{\varepsilon}\left\|v_{k-1}\right\|_{0, \rho^{-1}}\right)$.

Fix an $\varepsilon<1$ and take a $\lambda$ large enough so that $\left(\varepsilon+\frac{C(\varepsilon)}{\lambda-c}\right)<1$ also, then $\sum_{k=2}^{\infty}\left(\left\|v_{k}\right\|_{2, \rho}+\frac{C(\varepsilon)}{\varepsilon}\left\|v_{k}\right\|_{0, \rho^{-1}}\right)$ converges and this yields the convergency of $u_{k}(x)$ in $H^{2}(\Omega, \rho)$ and completes the proof.

Q.E.D.

\section{Appendix}

As was announced in the Introduction, we state here about mixed problems for hyperbolic equations.

Let us consider

$$
\left\{\begin{aligned}
& \frac{\partial^{2} u}{\partial t^{2}}= \rho(x)\left(\sum_{j, k=1}^{n} \frac{\partial}{\partial x_{j}}\left(a_{j k}(x) \frac{\partial u}{\partial x_{k}}\right)+2 \sum_{j=1}^{u} h_{j}(x) \frac{\partial^{2} u}{\partial x_{j} \partial t}+\sum_{j=1}^{n} b_{j}(x) \frac{\partial u}{\partial x_{j}}\right. \\
&\left.+h_{0}(x) \frac{\partial u}{\partial t}+c(x) u\right)+\sqrt{\rho(x)}\left(\sum_{j=1}^{n} p_{j}(x) \frac{\partial u}{\partial x_{j}}\right)+a_{0}(x) \frac{\partial u}{\partial t} \\
&+q(x) u+f(x, t) \\
& u(x, 0)=u_{0}(x), \frac{\partial}{\partial t} u(x, 0)=u_{1}(x) \\
&\left.u(x, t)\right|_{\Gamma}=0 \text { or } \frac{\partial u}{\partial \nu}-\sum_{j=1}^{n} h_{j}(x) \nu_{j} \frac{\partial u}{\partial t}+\left.\sigma u\right|_{\Gamma}=0,
\end{aligned}\right.
$$


where $h_{j}(x) \in \mathscr{B}^{1}(\Omega)(j=1, \ldots, n)$ and $\sigma \in \mathscr{B}^{1}(\Gamma)$ are all real-valued.

We assume that the rests of the coefficients satisfy the conditions stated in Introduction, then we can show the well-posedness of (A.1) in the following sense:

Theorem A.1. For any $f(x, t) \in \mathscr{E}_{t}^{1}\left(L^{2}\left(\Omega, \rho^{-1}\right)\right)$ and for any $\left(u_{0}, u_{1}\right)$ $\in H^{2}(\Omega, \rho) \cap \mathscr{D}_{L^{2}}^{1}(\Omega) \times \mathscr{D}{ }_{L^{2}}^{1}(\Omega)$ (for Dirichlet boundary condition) or for any $\left(u_{0}, u_{1}\right) \in H^{2}(\Omega, \rho) \cap \mathscr{N} \times H^{1}(\Omega)$ (for Neumann boundary condition), there exists a unique solution $u(x, t)$ of (A.1) such that

$$
u(x, t) \in \mathscr{E}_{t}^{0}\left(H^{2}(\Omega, \rho)\right) \cap \mathscr{E}_{t}^{1}\left(H^{1}(\Omega)\right) \cap \mathscr{E}_{t}^{2}\left(L^{2}\left(\Omega, \rho^{-1}\right)\right)
$$

with satisfying each boundary condition respectively. Moreover the following energy estimate holds in each case

$$
\begin{gathered}
\|u(t)\|_{2, \rho}+\left\|u_{t}(t)\right\|_{1}+\left\|u_{t t}(t)\right\|_{0, \rho^{-1}} \leqq C(T)\left(\left\|u_{0}\right\|_{2, \rho}+\left\|u_{1}\right\|_{1}+\right. \\
\left.+\|f(0)\|_{0, \rho^{-1}}+\int_{0}^{t}\left(\left\|f^{\prime}(s)\right\|_{0, \rho^{-1}}\right)+\left\|f^{\prime}(s)\right\|_{0, \rho^{-1}} d s\right)
\end{gathered}
$$

for any $t \in[0, T]$. Here $\mathcal{N}$ denotes

$$
\mathscr{N}=\left\{u ; u \in H^{2}(\Omega, \rho), \frac{\partial u}{\partial \nu}-\sum_{j=1}^{n} h_{j}(x) \nu_{j} \frac{\partial u}{\partial t}+\left.\sigma u\right|_{\Gamma}=0\right\}
$$

The proof of Theorem A.1 is carried out by the semi-group theory due to Hille-Yosida, and even for the equations with time dependent coefficients, the similar results will be valid.

\section{References}

[1] Agmon, S., A. Douglis, and L. Nirenberg, Estimates near the boundary for solutions of elliptic partial differential epuations, I, Comm. Pure Appl. Math. 12 (1959), 623-727.

[2] - Estimates near the boundary for solutions of elliptic partial differential equations, II, Comm. Pure Appl. Math. 17 (1964), 35-92.

[3] Lizorkin, P.I., Boundary properties of a certain class of functions, Dokl. Akad. Nauk SSSR 126 (1959), 103-106.

[4] - Boundary properties of functions of the "weight" classes, Dokl. Akad. Nauk SSSR 132 (1960), 514-517.

[5] Mizohata, S., Theory of Partial Differential Equations, Iwanami, Tokyo, 1965. 
(Japanese).

[6] Nakaoka, A., Boundary value problems for some degenerate elliptic equations of second order with Dirichlet condition, Proc. Japan Acad. 46 (1970), 248-252.

[7] - On Dirichlet problems for some degenerate elliptic equations, J. Math. Kyoto Univ. 10 (1970), 375-401.

[8] Nikol'skii, S.M., On imbedding, continuation and approximation theorems for differentiable functions of several variables, Rassian Math. Surveys, 16, No. 5 (1961), 55-104.

[9] Uspenskii, S.V., Imbedding theorem for class S.L. Sobolev of fractional order, Dokl. Akad. Nauk SSSR 130 (1960), 992-993.

[10] - Properties of classes $W_{p}^{(r)}$ with fractional derivative on differentiable manifolds, Dokl. Akad. Nauk SSSR 132 (1960), 60-62.

[11] Ikawa, M., Mixed problems for hyperbolic equations of second order, J. Math. Soc. Japan, 20 (1968), 580-608.

[12] Mizohata, S., Quelques problèmes au bord, du type mixte, pour des équations hyperboliques, Séminaire sur les équations aux derivées partielles, Collège de France (1966-1967), pp. 23-60.

[13] Yosida, K., On operator theoretical integration of wave equations, J. Math. Soc. Japan, 8 (1955), 649-675. 